\title{
COMPLETENESS OF NORMED LINEAR SPACES ADMITTING CENTERS
}

\author{
ALI ANSARI ASTANEH \\ (Received 2 February 1984) \\ Communicated by J. Chabrowski
}

\begin{abstract}
It is shown that a normed linear space admitting (Chebyshev) centers is complete. Then the ideas in the proof of this fact are used to show that every incomplete CLUR (compactly locally uniformly rotund) normed linear space contains a closed bounded convex subset $B$ with the following properties: (a) $B$ does not contain any farthest point; (b) $B$ does not contain any nearest point (to the elements of its complement).
\end{abstract}

1980 Mathematics subject classification (Amer. Math. Soc.): 46 B 20.

\section{Introduction}

Given a normed linear space $X$ and a bounded subset $A \subset X$, the farthest distance function $F_{A}: X \rightarrow R$ is defined by

$$
F_{A}(x)=\sup _{a \in A}\|x-a\|, \quad x \in X .
$$

Also, by definition, the Chebeshev radius of $A$ is defined by

$$
r(A)=\inf _{x \in X} F_{A}(x)
$$

The bounded subset $A$ is said to have a (Chebyshev) center if there exists some $c \in X$ such that $F_{A}(c)=r(A)$. The normed linear space $X$ is said to admit centers if each nonempty bounded subset in $X$ has at least one center. A. L. Garkavi (1964) has given sufficient conditions for a normed linear space to admit centers; these imply that all conjugate Banach spaces, and the spaces $L^{1}(\mu)$, admit centers. He has also established the fact that the uniform convexity of the norm in every

(C) 1985 Australian Mathematical Society $0263-6115 / 85 \$ A 2.00+0.00$ 
direction (see Garkavi (1964) for the definition) is a necessary and sufficient condition for a normed linear space to admit at most one center to every bounded subset. It is also known that the space $C_{R}(\Omega)$ of real-valued bounded continuous functions, on the paracompact topological space $\Omega$, admits centers (see Holmes (1972)). In the present paper we show that completeness is a necessary condition for a normed linear space to admit centers. This is done by constructing a bounded subset $B$ (in an incomplete normed linear space $X$ ) with no center. It is then shown (when assuming that $X$ is also CLUR) that the same subset $B$ serves as a closed bounded convex set in $X$ containing no farthest point and no nearest point. Since the closure of $B$ in the completion of $X$ is a closed ball, we naturally call $B$ a "virtual ball" in $X$.

\section{Results}

The following theorem implies that a normed linear space admitting centers must be complete.

THEOREM 1. Let $X$ be an incomplete normed linear space. Then $X$ contains a closed bounded and convex subset with no center.

Proof. Since $X$ is incomplete, it contains a Cauchy sequence $\left\{a_{n}\right\}$ which does not converge in norm. However for each $x \in X$, since

$$
\left\|x x-a_{n}\right\|-\left\|x-a_{m}\right\| \mid \leqslant\left\|a_{m}-a_{n}\right\|,
$$

and since the real line is complete, the sequence $\left\{\left\|x-a_{n}\right\|\right\}$ converges to a real number $r(x)$. We may assume without loss of generality that $r(0)=\lim _{n}\left\|a_{n}\right\|=1$. Observe that for each $\tilde{x}$ in the completion of $X$ we have $\lim _{n}\left\|\tilde{x}-a_{n}\right\|=$ $\left\|\tilde{x}-\lim _{n} a_{n}\right\|$, so that $r(x)$ equals the restriction of the continuous convex function $\left\|\tilde{x}-\lim _{n} a_{n}\right\|$ to $X$. It follows that $r(x)$ is also continuous and convex, and hence the subset $B=\{x \in X: r(x) \leqslant 1\}$ is a non-empty closed bounded and convex set in $X$. We show that $X$ does not admit a center to $B$. To this end it is enough to show that

(i) for each $x \in X, F_{B}(x)=\sup _{b \in B}\|x-b\|>1$, and

(ii) for each $\varepsilon>0$ there exists $a \in X$ with $F_{B}(a)<1+\varepsilon$.

To prove (i) let $x \in X$ be arbitrary. Then $0<r(x)$ (otherwise $a_{n} \rightarrow x$ which is absurd). Now from $\lim _{n}\left\|x-a_{n}\right\|=r(x)$ and $\lim _{m, n}\left\|a_{m}-a_{n}\right\|=0$ we can choose a natural number $N$ such that

$$
\forall n>N, \quad\left\|a_{N}-a_{n}\right\|<\frac{r(x)}{M}<\left\|x-a_{N}\right\|,
$$


where $M$ is some fixed positive number for which $r(x) / M<1<M$. Next we put $b=a_{N}+\left(\left(a_{N}-x\right) /\left\|a_{N}-x\right\|\right)(1-r(x) / M)$, and consider that by (1)

$$
\forall n>N, \quad\left\|b-a_{n}\right\| \leqslant\left\|b-a_{N}\right\|+\left\|a_{N}-a_{n}\right\|=1-\frac{r(x)}{M}=\left\|a_{N}-a_{n}\right\|<1,
$$

from which it follows $r(b) \leqslant 1$ and hence $b \in B$. Now, again by (1),

$$
\begin{aligned}
\|x-b\| & =\left\|x-a_{N}-\left(\left(a_{N}-x\right) /\left\|a_{N}-x\right\|\right)(1-r(x) / M)\right\| \\
& =\left\|x-a_{N}\right\|+1-r(x) / M>1,
\end{aligned}
$$

from which we deduce $F_{B}(x) \geqslant\|x-b\|>1$ and (i) is proved.

To show (ii) let $0<\varepsilon$ be arbitrary. Since $\left\{a_{n}\right\}$ is Cauchy we first choose a natural number $N$ such that for $n>N,\left\|a_{N}-a_{n}\right\|<\varepsilon / 4$.

We show that $F_{B}\left(a_{N}\right)<1+\varepsilon$ : given an arbitrary $b \in B, r(b) \leqslant 1$ implies the existence of an $n>N$ such that $\left\|b-a_{n}\right\|<1+\varepsilon / 4$. Hence

$$
\left\|a_{N}-b\right\| \leqslant\left\|a_{N}-a_{n}\right\|+\left\|a_{n}-b\right\|<\varepsilon / 4+1+\varepsilon / 4 .
$$

Therefore for each $b \in B$ we get $\left\|a_{N}-b\right\|<1+\varepsilon / 2$ and hence $F_{B}\left(a_{N}\right)<1+\varepsilon$. This completes the proof of the theorem.

We naturally propose to call the set $B$, constructed in the proof of Theorem 1 , a "virtual ball", as it is the intersection with $X$ of the ball $B\left(\lim _{n} a_{n}, 1\right)$ in the completion of $X$. Our next theorem shows that in an incomplete CLUR space, a virtual ball contains neither a farthest point (to any element of $X$ ), nor a nearest point (to any element of its complement). Recall that a normed linear space $X$ is called CLUR (or $X$ is said to have property $(M)$ in the terminology of Panda and Kapoor (1975)); if $x, x_{n} \in X,\|x\|=1,\left\|x_{n}\right\| \leqslant 1$, and $\lim _{n}\left\|x_{n}+x\right\|=2$ implies that $\left\{x_{n}\right\}$ has a convergent subsequence. The CLUR normed linear spaces were first introduced by L. P. Vlasov (1967), and were studied in some detail by Panda and Kapoor (1975). In the proof of our Theorem 2 we shall make use of the following lemma. The two parts of this lemma are modified versions of Lemma 1(ii) and Lemma 2(ii) in Astaneh (1983). As the second implication was stated there without a proof, and for the sake of completeness, we present here a short proof.

LEMMA. Let $X$ be a CLUR normed linear space.

(i) Given any closed ball $B(z, r)$ and $x(\neq z) \in X$, each maximizing sequence for $x$ in $B(z, r)$ has a convergent subsequence.

(ii) Given any open ball $B^{0}(z, r)$ and any $x(\neq z) \in B^{0}(z, r)$, each minimizing sequence for $x$ in $X \sim B^{0}(z, r)$ has a convergent subsequence $\left(X \sim B^{0}(z, r)\right.$ denotes the complement of $B^{0}(z, r)$ in $\left.X\right)$. 
Proof. (i) Let $x$ and $B(z, r)$ be as stated and let $\left\{y_{n}\right\}$ be a maximizing sequence for $x$ in $B(z, r)$. Without loss of generality we may translate and scale so that $x=0$ and $r+\|z\|=1$, in which case we will have $\left\|y_{n}\right\| \rightarrow 1$. Then for each $n$,

$$
\begin{aligned}
2 & \geqslant\left\|y_{n}\right\|+\left\|\frac{z}{1-r}\right\| \geqslant\left\|y_{n}+\frac{z}{1-r}\right\|=\left\|\frac{2-r}{1-r} y_{n}-\frac{1}{1-r}\left(y_{n}-z\right)\right\| \\
& \geqslant \frac{2-r}{1-r}\left\|y_{n}\right\|-\frac{1}{1-r}\left\|y_{n}-z\right\| \rightarrow \frac{2-r}{1-r}+\frac{r}{1-r}=2 .
\end{aligned}
$$

Therefore $\left\|y_{n}+z /(1-r)\right\| \rightarrow 2$, and by the CLUR property of $X,\left\{y_{n}\right\}$ has a convergent subsequence.

(ii) Let $x$ and $B^{0}(z, r)$ be as stated and let $\left\{y_{n}\right\}$ be a minimizing sequence for $x$ in $X \sim B^{0}(z, r)$. Again without loss of generality we may translate and scale, but this time in such a way that $z=0$ and $r=1$. Then $\left\|y_{n}\right\| \geqslant 1$ and $\left\|y_{n}\right\| \rightarrow 1$, and hence

$$
\begin{aligned}
2 & \geqslant\left\|\frac{x}{\|x\|}+\frac{y_{n}}{\left\|y_{n}\right\|}\right\|=\left\|\frac{1}{\|x\|}\left(x-y_{n}\right)+\left(\frac{1}{\|x\|}+\frac{1}{\left\|y_{n}\right\|}\right) y_{n}\right\| \\
& \geqslant\left\|y_{n}\right\|\left(\frac{1}{\|x\|}+\frac{1}{\left\|y_{n}\right\|}\right)-\frac{1}{\|x\|}\left\|x-y_{n}\right\| \\
& =\frac{1}{\|x\|}\left(\left\|y_{n}\right\|-\left\|x-y_{n}\right\|\right)+1 \rightarrow \frac{1}{\|x\|}[1-(1-\|x\|)]+1=2 .
\end{aligned}
$$

Therefore

$$
\left\|\frac{x}{\|x\|}+\frac{y_{n}}{\left\|y_{n}\right\|}\right\| \rightarrow 2
$$

and, $X$ being CLUR, $\left\{y_{n} /\left\|y_{n}\right\|\right\}$ has a convergent subsequence. From this and $\left\|y_{n}\right\| \rightarrow 1$ the result follows.

THEOREM 2. Let $X$ be an incomplete CLUR normed linear space. Then any virtual ball $B$ in $X$ is a closed bounded convex subset with the following properties:

(i) $B$ does not admit a nearest point to any element of its coplement;

(ii) $B$ does not admit a farthest point to any element of $X$.

Proof. (i) Let $B$ be the set constructed in Theorem 1. We show that no element belonging to the complement of $B$ has a nearest point in $B$. To this end let $x \notin B$; then $r(x)>1$ and therefore $r(x)-1>0$. We first note that

$$
\inf _{b \in B}\|x-b\|=r(x)-1 .
$$


To show (1) we only need to consider the ball $B\left(\lim _{n} a_{n}, 1\right)$ in the completion of $X$, and note that $B$ is dense in this ball. Then, having observed this, we have

$$
\begin{aligned}
\inf _{b \in B}\|x-b\| & =\inf \left\{\|x-\tilde{b}\|: \tilde{b} \in B\left(\lim _{n} a_{n}, 1\right)\right\} \\
& =\left\|x-\lim _{n} a_{n}\right\|-1=r(x)-1 .
\end{aligned}
$$

To show that $B$ does not admit a nearest point to $x$ we assume otherwise, and get a contradiction. Thus let $b_{0} \in B$ and

$$
\left\|x-b_{0}\right\|=\inf _{b \in B}\|x-b\|=r(x)-1 .
$$

Since $b_{0}$ must belong to the boundary of $B$, by the definition of $B$ we have $r\left(b_{0}\right)=\lim _{n}\left\|b_{0}-a_{n}\right\|=1$. We next consider the ball $B\left(b_{0}, 1\right)$ and note that the sequence $a_{n}^{\prime}=b_{0}+\left(a_{n}-b_{0}\right) /\left\|z_{n}-b_{0}\right\|$ lies on the boundary of $B\left(b_{0}, 1\right)$, since $\left\|a_{n}^{\prime}-b_{0}\right\|=1$. On the other hand

$$
\lim _{n}\left\|a_{n}^{\prime}-a_{n}\right\|=\lim _{n} \frac{\left(\left\|a_{n}-b_{0}\right\|-1\right)\left\|a_{n}-b_{0}\right\|}{\left\|a_{n}-b_{0}\right\|}=0 .
$$

From (2) and $\lim _{n}\left\|x-a_{n}\right\|=r(x)$, it follows that $\lim _{n}\left\|x-a_{n}^{\prime}\right\|=r(x)$. Now considering $a_{n}^{\prime} \in B\left(b_{0}, 1\right)$ and the fact that $r(x)=\left\|x-b_{0}\right\|+1$, we deduce that $\left\{a_{n}^{\prime}\right\}$ is a maximizing sequence for $x$ in $B\left(b_{0}, 1\right)$. Therefore, noting $X$ is CLUR and by part (i) of the above Lemma, we see that $\left\{a_{n}^{\prime}\right\}$ must have a convergent subsequence. But (since $a_{n}=\left\|a_{n}-b_{0}\right\|\left(a_{n}^{\prime}-b_{0}\right)+b_{0}$ ), this would imply that $\left\{a_{n}\right\}$ has a convergent subsequence, which is a contradiction. Hence the proof is complete.

(ii) Let $x \in X$ be arbitrary. We first note that

$$
\sup _{b \in B}\|x-b\|=r(x)+1 .
$$

To show (3) it is again enough to consider the ball $B\left(\lim _{n} a_{n}, 1\right)$ in the completion of $X$, and that $B$ is dense in this ball, so that

$$
\sup _{b \in B}\|x-b\|=\sup \left\{\|x-\tilde{b}\|: \tilde{b} \in B\left(\lim _{n} a_{n}, 1\right)\right\}=\left\|x-\lim _{n} a_{n}\right\|+1 .
$$

Hence

$$
\sup _{b \in B}\|x-b\|=r(x)+1 .
$$

To show $B$ does not admit a farthest point to $x$ we assume otherwise. Thus suppose $c \in B$ is such that $\|x-c\|=\sup _{b \in B}\|x-b\|=r(x)+1$. Let $z=x+$ $(x-c) /\|x-c\|$, and also for each $n$ let $a_{n}^{\prime}=c+\left(a_{n}-c\right) /\left\|a_{n}-c\right\|$. As in the proof of (i) we have $\lim _{n}\left\|a_{n}^{\prime}-a_{n}\right\|=0$ and therefore $\lim _{n}\left\|x-a_{n}^{\prime}\right\|=r(x)$. We 
next consider that

$$
\begin{aligned}
\left\|z-a_{n}^{\prime}\right\| & =\left\|x+\frac{x-c}{\|x-c\|}-c-\frac{a_{n}-c}{\left\|a_{n}-c\right\|}\right\| \\
& =\left\|\left(1+\frac{1}{\|x-c\|}\right)(x-c)-\frac{a_{n}-c}{\left\|a_{n}-c\right\|}\right\| \\
& \geqslant\left\|\left(1+\frac{1}{\|x-c\|}\right)(x-c)\right\|-1=(\|x-c\|+1)-1=r(x)+1 .
\end{aligned}
$$

This means that $a_{n}^{\prime} \in X \sim B^{0}(z, r(x)+1)$. On the other hand

$$
\inf \left\{\|x-y\|: y \in X \sim B^{0}(z, r(x)+1)\right\}=r(x)+1-\|x-z\|=r(x) .
$$

Hence $\left\{a_{n}^{\prime}\right\}$ is a minimizing sequence for $x \in B^{0}(z, r(x)+1)$ in $X \sim B^{0}(z, r(x)$ +1 ). Now since $x \neq z$ by part (ii) of the above lemma $\left\{a_{n}^{\prime}\right\}$ must have a convergent subsequence. But then (by $\lim _{n}\left\|a_{n}^{\prime}-a_{n}\right\|=0$ ) this would mean that $\left\{a_{n}\right\}$ has a convergent subsequence in $X$, which is absurd. Hence (ii) follows.

ExAmple. Consider the normed linear space $\left(X,\|\|_{2}\right)$ where $X=C([-1,1])$ is the space of continuous functions on $[-1,1]$ and $\|x\|_{2}=\left(\int_{-1}^{1}|x(t)|^{2} d t\right)^{1 / 2}$. Being an inner product space, $X$ is uniformly convex and hence a CLUR normed linear space. The Cauchy sequence

$$
a_{n}(t)= \begin{cases}0, & -1 \leqslant t \leqslant 0, \\ n t, & 0<t<\frac{1}{n}, \\ 1, & \frac{1}{n} \leqslant t \leqslant 1,\end{cases}
$$

does not converge in $\left(X,\|\|_{2}\right)$, though in the completion $\tilde{X}\left(=L^{2}([-1,1])\right)$ of $X$ it converges to $\chi_{(0,1]}$ (the characteristic function of $\left.(0,1]\right)$. Now it follows from Theorem 1 that

$$
\begin{aligned}
B & =\left\{x \in X: \| x-\chi_{\left.(0,1] \|_{2} \leqslant 1\right\}}\right. \\
& =\left\{x \in C([-1,1]): \int_{-1}^{0}|x(t)|^{2} d t+\int_{0}^{1}|x(t)-1|^{2} d t \leqslant 1\right\}
\end{aligned}
$$

is a closed bounded convex subset with no (Chebyshev) center. Moreover by Theorem 2, $B$ contains neither a nearest point (to the elements of its complement), nor a farthest point.

\section{Acknowledgement}

The author wishes to thank the referee for his valuable suggestions, which simplified several arguments in the original manuscript, particularly for pointing out an error (by means of a counterexample) which occured in the original proof 
of part (ii) of Theorem 2. The short proof of part (i) of the lemma is due to the referee, who kindly suggested including such a proof, rather than giving references.

The author also thanks his colleague Dr. M. A. Pour Abdollah for valuable discussions.

\section{References}

A. A. Astaneh (1983), 'A characterization of local uniform convexity of the norm', Indian J. Pure Appl. Math. 14 (10), 1217-1219.

A. L. Garkavi (1964), 'The best possible net and the best possible cross section of a set in a normed linear space', Amer. Math. Soc. Transl. Ser. 2, 39, 111-132.

R. B. Holmes (1972) A course in optimization and best approximation, (Lecture Notes in Math., vol. 257, Springer-Verlag, New York, London).

B. B. Panda and O. P. Kapoor (1975), 'A generalization of local uniform convexity of the norm', $J$. Math. Anal. Appl. 52, 300-308.

\section{Department of Mathematics}

University of Mashhad

Mashhad, Iran 